\title{
Estimating Dietary Protein Intake in Peritoneal Dialysis Patients: The Effect of Ethnicity
}

Kidney dialysis patients with sarcopenia have increased mortality. Clinical guidelines recommend peritoneal dialysis (PD) patients have a target daily protein intake to prevent sarcopenia. Protein intake is estimated from total daily urea losses in urine and peritoneal dialysate to assess the protein equivalent of nitrogen appearance rate adjusted for body weight (nPNA). Dietary habits differ among ethnic groups, so we reviewed nPNA and body composition in a multi-ethnic PD population. Body composition was measured with multifrequency bioimpedance in 598 patients (301 white, 136 black, 123 South-Asian, and 38 Asian-Pacific). South-Asians had a lower $n$ PNA compared with white and black individuals (Randerson $0.80 \pm 0.21$ vs $0.88 \pm 0.24$ and $0.85 \pm 0.24 \mathrm{~g} / \mathrm{kg}$ / day, Blumenkrantz $0.97 \pm 0.14$ vs $1.04 \pm 0.22$ and $0.99 \pm 0.22 \mathrm{~g} / \mathrm{kg} /$ day, Bergström $0.87 \pm 0.4$ vs $0.95 \pm 0.24$ and $0.92 \pm 0.24 \mathrm{~g} / \mathrm{kg} / \mathrm{day}$ all $p<0.001)$. South-Asians had lower weights $(68.9 \pm 14.9$ vs $74.4 \pm 16.6$ and $73.5 \pm 16.3 \mathrm{~kg}, p<0.001$ ), and although of similar body mass index $\left(25.9 \pm 4.9 \mathrm{vs} 28.5 \pm 4.9\right.$ and $\left.26.5 \pm 5.2 \mathrm{~kg} / \mathrm{m}^{2}\right)$, had both lower skeletal muscle and appendicular muscle mass indexed for height $(9.08 \pm 1.45$ vs $9.89 \pm 1.62$ and $10.1 \pm 1.85$, $p<0.001$; and $6.95 \pm 1.39$ vs $7.68 \pm 1.48$ and $7.67 \pm 1.58 \mathrm{~kg} / \mathrm{m}^{2}$ $p<0.01)$. South-Asian patients had a lower calculated basal metabolic rate (BMR) $(1,358 \pm 218$ vs $1,487 \pm 257$ and $1,489 \pm$ $271 \mathrm{kcal} /$ day, $p<0.001)$.

Asian PD patients, particularly South-Asians, have lower dietary protein intakes when calculated by nPNA. However, South-Asians had lower measured muscle mass and calculated BMR. As such, dietary protein intake targets derived from studies in 1 ethnic group are not necessarily applicable for all patients, as those with less muscle mass and lower BMR may well need less daily protein intake to maintain homeostasis.

Perit Dial Int 2018; 38(5):384-387

www.PDIConnect.com https://doi.org/10.3747/pdi.2017.00260

KEY WORDS: Muscle; protein nitrogen; nutrition; bioimpedance.

Patie atients with chronic kidney disease are at increased risk of muscle wasting, or sarcopenia (1). In addition to urinary protein losses, peritoneal dialysis (PD) patients also have protein losses in the spent dialysate (2). As such, clinical guidelines recommend regular assessment of dietary protein intake, along with targets for protein intake $(\geq 1.2 \mathrm{~g} / \mathrm{kg} /$ day), to prevent malnutrition and sarcopenia (3). Although dietary protein intake can be estimated by food diaries and dietary recall, clinical guidelines recommend using equations to calculate a protein equivalent of nitrogen appearance (PNA) based on urinary and peritoneal urea losses $(3,4)$. 
Dietary preferences and body composition may differ among patients from different ethnic groups (5). We wished to determine whether there were differences in the estimates of dietary protein intake with ethnicity between the 3 most commonly used equations (6).

\section{METHODS}

We measured body composition using multifrequency bioelectrical impedance (MFBIA) (InBody 720, Seoul, South Korea) (7). Patients had not had peritonitis or other infections, or hospital admissions within the previous 8 weeks. Multifrequency bioelectrical impedance (8) was measured in a previously reported standardized manner; first patients were asked to empty their bladders, and then peritoneal dialysate was drained out, as ascites and peritoneal dialysate can potentially alter bioimpedance-derived body composition measurements (9). We used the manufacturer's software algorithm to estimate body composition, which we have previously reported correlated with dual electron absorptiometry in dialysis patients $(10,11)$. Basal metabolic rate (BMR) was estimated from the Cunningham equation (12). Patients with amputations, pregnancy, and those with limb paralysis were excluded. Patient demographics and ethnicity were obtained from the hospital's computerized records.

Urea was measured by standard laboratory methods (Roche Integra; Roche diagnostics, Lewes, UK) in 24-hour urine collections and each bag of spent peritoneal dialysate. The PNA was calculated by recommended equations: Randerson, Bergström, and Blemenkrantz (6). To compare patients, PNA was adjusted for body weight (nPNA) and body composition indexed to height.

This retrospective audit complied with the UK National Health Service (NHS) guidelines for clinical audit and service development. All patient data were anonymized.

\section{STATISTICAL ANALYSIS}

Data are presented as mean \pm standard deviation, median (interquartile range), or percentage. Standard statistical tests (ANOVA, Kruskal Wallis, or Chi-squared test) were applied, with appropriate post hoc corrections made for multiple testing (Tukey or Dunn), where appropriate. Statistical analysis used Prism 7.0 (Graph Pad, San Diego, CA, USA) and SPSS 24 (IBM Corporation, Armonk, New York, USA). Statistical significance was taken as $p<0.05$.

\section{RESULTS}

We compared nPNA in 598 PD patients at the time of their first assessment of peritoneal membrane function, and excluded 4 due to mixed ethnicity (Table 1). Most patients were of white ethnicity, and fewest were Asian-Pacific, who were also the youngest. Asian patients had lower weight, muscle mass, and muscle mass indexed for height, particularly in the South Asians (Table 1). Although PD adequacy and urine output were similar, South Asians had a lower nPNA when calculated with all 3 equations (Figure 1). When BMR was calculated from MFBIA body composition (12), South Asians had a lower BMR.

\section{DISCUSSION}

Dialysis patients with sarcopenia are at increased risk of mortality (3), and unlike fat, there is no protein reserve store. So, failure to meet nutritional targets potentially risks muscle breakdown and loss. In routine clinical practice, nutritional targets, in terms of protein intake, are based on urea removed $(3,4)$. Although PNA is widely adopted, the studies on which the equations for calculating PNA are based included only a small number of patients, who were treated with continuous ambulatory PD (CAPD) (6). Previous reports have shown that there are differences between the 3 most commonly used equations, depending upon the patient group studied, and there is no consensus as to whether any 1 of these equations has superiority. Certainly, in Europe and North America, the demographics of patients treated with PD has changed since these studies were performed several decades ago, and more patients are now treated using cyclers.

We used MFBIA, which has been validated with studies of dual energy X-ray absorptiometry $(10,11)$ to determine body composition. Our findings demonstrate that in patients recently started on $\mathrm{PD}$, despite similar over-all clearance, estimates of PNA adjusted for body weight were lower in South Asian patients than in white and black ethnic groups with all 3 of the commonly used equations. Asian patients were of lower overall weight, and the South Asian patients in particular had lower total and appendicular muscle mass. In addition, calculation of BMR (12) showed that South Asians had a lower BMR. It has been suggested that the amount of dialysis a patient requires should be based on metabolic rate, as this reflects cellular activity and production of waste products of metabolism. Recalculating dialysis adequacy using this concept rather than total body water derived from anthropomorphic equations shows that patients with greater muscle mass have a higher metabolic rate and require more dialysis clearance (13). Equally, patients with less muscle mass have lower energy expenditure, and so not only have lower cellular metabolism but would also be expected to require lower protein intake to maintain homeostasis. Therefore, if all patients were dialyzed to achieve the same weekly urea clearance target, then the patient with lower BMR and muscle mass would receive a relatively greater dialysis dose. Interestingly, an observational report from Canada noted better survival for Asian PD patients than other ethnicities (14).

We have performed a cross-sectional analysis, and there are a number of limitations to be considered, such as the assumption that patients were in a neutral nitrogen balance, and the fact that the different ethnic groups were not casematched. We estimated PNA and BMR rather than performing formal studies.

However, our data would suggest that nutritional targets for protein intake in white and black ethnic groups with chronic 
TABLE 1

Demographics, Dialysis Prescriptions, Body Composition and Laboratory Investigations in Patients According to Ethnicity

\begin{tabular}{|c|c|c|c|c|}
\hline & White & Black & South Asian & Asian-Pacific \\
\hline Number & 301 & 136 & 123 & 38 \\
\hline Male & $181(59.7)$ & $55(40.4)^{a}$ & $74(60.2)$ & $22(56.4)$ \\
\hline Age, years & $58.6 \pm 17.0$ & $50.4 \pm 14.4$ & $58.0 \pm 15.0$ & $40.3 \pm 7.3^{a}$ \\
\hline Weight, kg & $74.4 \pm 16.6^{c}$ & $73.5 \pm 16.3^{c}$ & $68.9 \pm 14.1$ & $65.6 \pm 9.3$ \\
\hline $\mathrm{BMI} \mathrm{kg} / \mathrm{m}^{2}$ & $28.5 \pm 4.9$ & $26.5 \pm 5.2$ & $25.9 \pm 4.9$ & $25.9 \pm 3.5$ \\
\hline SLM, kg & $49.9 \pm 11.0^{c}$ & $49.6 \pm 11.7^{c}$ & $44.4 \pm 9.8$ & $46.7 \pm 6.3$ \\
\hline SMM, kg & $28.0 \pm 6.9^{c}$ & $28.2 \pm 7.5^{c}$ & $24.5 \pm 5.7$ & $25.2 \pm 6.4$ \\
\hline $\mathrm{BF}, \mathrm{kg}$ & $22.7 \pm 10.8$ & $21.9 \pm 11.3$ & $23.0 \pm 10.3$ & $21.5 \pm 8.5$ \\
\hline Append, kg & $21.7 \pm 6.0^{c}$ & $21.7 \pm 6.4^{c}$ & $18.9 \pm 5.2$ & $21.0 \pm 4.0$ \\
\hline SMMI, $\mathrm{kg} / \mathrm{m}^{2}$ & $9.89 \pm 1.62^{c}$ & $10.1 \pm 1.85^{c}$ & $9.08 \pm 1.45$ & $9.45 \pm 1.58$ \\
\hline AppendI, kg/m² & $7.68 \pm 1.48^{b}$ & $7.67 \pm 1.58^{b}$ & $6.95 \pm 1.39$ & $7.14 \pm 1.38$ \\
\hline BMR, kcal/day & $1,487 \pm 257^{c}$ & $1,489 \pm 271^{c}$ & $1,358 \pm 123$ & $1,439 \pm 112$ \\
\hline Hemoglobin, g/dL & $11.3 \pm 1.7$ & $11.0 \pm 1.4$ & $11.3 \pm 1.5$ & $11.7 \pm 1.5$ \\
\hline Albumin, $\mathrm{g} / \mathrm{L}$ & $37.6 \pm 4.6$ & $37.8 \pm 4.9$ & $37.7 \pm 9.2$ & $37.0 \pm 5.2$ \\
\hline PD vintage, mo & $3(2-12)$ & $2(2-5.5)$ & $2(2-9)$ & $3(2-17)$ \\
\hline CAPD & $82(27.2)$ & $19(14)$ & $32(26.0)$ & $7(18.4)$ \\
\hline Icodextrin & $217(72.6)$ & $107(79.7)$ & $88(72.1)$ & $31(81.6)$ \\
\hline $22.7 \%$ glucose & $92(30.8)$ & $43(31.6)$ & $34(27.9)$ & $16(42.1)$ \\
\hline Urine $\mathrm{mL} /$ day & $1,124(532-1,658)$ & $972(475-1,505)$ & $1,023(528-1,595)$ & $917(565-1,627)$ \\
\hline Weekly Kt/Vurea & $2.76(2.05-3.49)$ & $2.67(2.0-3.3)$ & $2.7(2.24-3.49)$ & $2.13(1.98-3.93)$ \\
\hline 4-hr D/Screatinine & $0.73 \pm 0.13$ & $0.72 \pm 0.14$ & $0.72 \pm 0.13$ & $0.67 \pm 0.18$ \\
\hline
\end{tabular}

BMI = body mass index; SLM = soft lean mass; SMM = skeletal muscle mass; $B F=$ body fat; $A$ ppend = appendicular muscle mass; SMMI = SMM indexed to height; $A$ ppendI = appendicular muscle mass indexed to height; $B M R=$ basal metabolic rate; $P D=$ peritoneal dialysis; $C A P D=$ continuous ambulatory PD; 4-h D/Screatinine = 4-hour peritoneal equilibration test dialysate to serum creatinine.

Results expressed as integers, mean \pm standard deviation, median (interquartile range) or $n$ (percentage).

a $p<0.05$ vs South Asian patients.

${ }^{\mathrm{b}} p<0.01$ vs South Asian patients.

${ }^{\mathrm{c}} p<0.001$ vs South Asian patients.

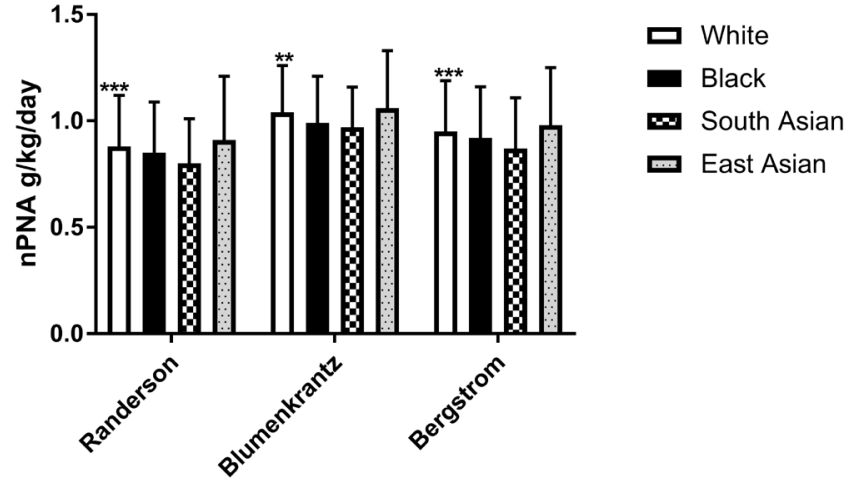

Figure 1 - Protein equivalent of nitrogen appearance adjusted for body weight (nPNA) according to ethnicity. ${ }^{* *} p<0.01,{ }^{* * *} p<0.001$ vs South Asian patients.

kidney disease living in European and North America and treated with PD do not readily translate to other ethnic groups, particularly South Asians. We were only able to study a very small number of Asian-Pacific patients, and although they had lower weight and overall muscle mass, when muscle mass was indexed for height it was not different from the white patients. However, this was a younger group, and muscle mass declines with age (1). As such, it is likely that our findings would also apply to older matched Asian-Pacific PD patients.

Our preliminary data would suggest that dietary protein intake does vary among ethnic groups, as this was a consistent finding with all 3 equations used to estimate PNA (6). Those patients with lower BMR and PNA have lower muscle mass and may therefore potentially have a slower generation of uremic toxins. This could suggest a link between the amount of dialysis individual patients should receive and their nitrogen turnover and may provide an explanation for better patient survival for Asian dialysis patients compared with those from other ethnicities. Our results do not suggest that patients with lower muscle mass and dietary protein intake may require less dialysis, but rather that, speculating from observational reports, the delivery of more dialysis to these patients is associated with better survival. Our study potentially generates a number of hypotheses on several levels, which will require future study.

\section{ACKNOWLEDGMENTS}

Dr. Surachet Vongsanim was in receipt of an International Society of Nephrology training scholarship. 


\title{
DISCLOSURES
}

The authors have no financial conflicts of interest to declare.

\author{
Surachet Vongsanim ${ }^{1}$ \\ Andrew Davenport ${ }^{2 *}$ \\ Renal Division ${ }^{1}$ \\ Department of Internal Medicine \\ Chiang Mai University Hospital \\ Chiang Mai, Thailand \\ UCL Centre for Nephrology ${ }^{2}$ \\ Royal Free Hospital \\ University College London Medical School \\ London, United Kingdom \\ *email: andrewdavenport@nhs.net
}

\section{REFERENCES}

1. Fahal IH. Uraemic sarcopenia: aetiology and implications. Nephrol Dial Transplant 2014; 29(9):1655-65.

2. Rajakaruna G, Caplin B, Davenport A. Peritoneal protein clearance rather than faster transport status determines outcomes in peritoneal dialysis patients. Perit Dial Int 2015; 35(2):216-21.

3. Dombros N, Dratwa M, Feriani M, Gokal R, Heimbürger 0, Krediet R, EBPG Expert Group on Peritoneal Dialysis, et al. European best practice guidelines for peritoneal dialysis. 8 Nutrition in peritoneal dialysis. Nephrol Dial Transplant 2005; 20(Suppl 9):ix28-33.

4. NKF-DOQI clinical practice guidelines for peritoneal dialysis adequacy. Assessment of nutritional status. Am J Kidney Dis 2007; 30(3 Suppl 2):S125-9.

5. Davenport A, Hussain Sayed R, Fan S. The effect of racial origin on total body water volume in peritoneal dialysis patients. Clin J Am Soc Nephrol 2011; 6(10):2492-8.

6. Bergström J, Heimbürger 0, Lindholm B. Calculation of the protein equivalent of total nitrogen appearance from urea appearance. Which formulas should be used? Perit Dial Int 1998; 18:467-73.

7. Davies SJ, Davenport A. The role of bioimpedance and biomarkers in helping to aid clinical decision-making of volume assessments in dialysis patients. Kidney Int 2014; 86(3):489-96.

8. Davenport A, Willicombe M. Comparison of fluid status in patients treated by different modalities of peritoneal dialysis using multi-frequency bioimpedance. Int J Artif Organs 2009; 32(11):779-86.

9. Davenport A. Effect of intra-abdominal dialysate on bioimpedance-derived fluid volume status and body composition measurements in peritoneal dialysis patients. Perit Dial Int 2013; 33(5):578-9.

10. Fürstenberg A, Davenport A. Assessment of body composition in peritoneal dialysis patients using bioelectrical impedance and dual-energy $\mathrm{x}$-ray absorptiometry. Am J Nephrol 2011; 33(2):150-6.

11. Fürstenberg A, Davenport A. Comparison of multifrequency bioelectrical impedance analysis and dual-energy $\mathrm{X}$-ray absorptiometry assessments in outpatient haemodialysis patients. Am J Kidney Dis 2011; 57(1):123-9.

12. El-Kateb S, Sridharan S, Farrington K, Fan S, Davenport A. Comparison of equations of resting and total energy expenditure in peritoneal dialysis patients using body composition measurements determined by multifrequency bioimpedance. Clin Nutr 2018; 37(2):646-50.

13. El-Kateb S, Sridharan S, Farrington K, Fan S, Davenport A. A single weekly $\mathrm{Kt} /$ Vurea target for peritoneal dialysis patients does not provide an equal dialysis dose for all. Kidney Int 2016; 90(6):1342-7.

14. Hemmelgarn BR, Chou S, Wiebe N, Culleton BF, Manns BJ, Klarenbach S, et al. Differences in use of peritoneal dialysis and survival among East Asian, Indo Asian, and white ESRD patients in Canada. Am J Kidney Dis 2006; 48(6):964-71.

The single copy is for your personal, non-commercial use only. For permission to reprint multiple copies or to order presentation-ready copies for distribution, contact Multimed Inc. at marketing@multi-med.com 